\title{
Gravitational Telescope
}

\author{
Alexander V. Lukanenkov \\ Moscow, Russia \\ Email: a_v_luk@mail.ru \\ Received 13 January 2016; accepted 9 April 2016; published 13 April 2016 \\ Copyright (C) 2016 by author and Scientific Research Publishing Inc. \\ This work is licensed under the Creative Commons Attribution International License (CC BY). \\ http://creativecommons.org/licenses/by/4.0/ \\ (c) (i) Open Access
}

\begin{abstract}
It's proposed to use a global seismic antenna (GSA) as a gravitational telescope, arbitrary "quiet" seismic stations are its elements, and aperture of GSA must be of the order $10,000 \mathrm{~km}$. The relative displacements of various points of the Earth are detected by GSA, these displacements are described as quasi-harmonic elliptical signals generated by gravitational waves, their amplitude $\approx$ $2.5 \times 10^{-15} \mathrm{~m}$. It is found that these waves cause deformation (strain) of the order $h \approx 10^{-21}$. Pulsars are a natural source of periodic waves. The fact of confident registration of gravitational wave is confirmed by detection of quasi-harmonic signals in the frequency band near $6.023 \mathrm{~Hz}$ for 90 hours (confidence probability of detection is close to 1). It is found that a small part of the rotation energy of associated pulsar $\left(\varepsilon \approx 1^{-5}\right)$ is expended on the radiation corresponding to the gravitational wave.
\end{abstract}

\section{Keywords}

Gravitational Waves, Gravitational Signal, Gravitational Telescope, Seismic Antenna, Deformation, Optimal Signal Processing

\section{Introduction}

Gravitational waves (GW-waves) are the inevitable consequence of many theories of gravity [1] [2]. Astrophysical observations indicate their existence. Indirectly, gravitational waves have been identified in the motion of binary pulsars [3].

Gravitational telescopes (gravitational antennas) are created for the direct detection of gravitational waves. There are two types of gravitational wave detectors [4].

The low-frequency mechanical vibrations of a massive body caused by gravitational wave are measured in the first type. Detectors of this type use a massive metal bar, cooled to a low temperature. Weber bar is a famous example of such detector.

From the currently valid detectors on this principle operate spherical antenna MiniGRAIL, as well as antenna ALLEGRO, AURIGA, EXPLORER and NAUTILUS. 
Detector of another type uses laser interferometry to measure gravitational wave induced motion between separated "free" masses.

This principle is applied in experiments LIGO, GEO600, TAMA-300 and VIRGO.

There are also projects for the detection of gravitational waves using seismographs [4]-[6].

It's proposed to use a global seismic antenna (GSA) as a gravitational telescope, and arbitrary "quiet" seismic stations are its elements, its aperture must be of the order $10,000 \mathrm{~km}$.

Demonstration of the GSA possibilities was carried by the results of registration of gravitational radiation at a frequency $f \approx 6 \mathrm{~Hz}$.

\section{The Required Threshold Sensitivity of GSA}

General relativity (GR) predicts the existence of gravitational radiation (GW-radiation) as a perturbation of the gravitational field.

The corresponding gravitational wave (GW-wave) moves at the speed of light and is described by two independent components, arranged at an angle $45^{\circ}$ to each other (Figure 1).

The simplest type: periodic compression and stretching of the body in two antiphase directions (Figure 1) [7], i.e. the body under the action of waves is slightly compressed and stretched in two horizontal directions, and compression and stretching will be swapped after half period.

GW-wave leads to a relative deformation of the body, therefore, the absolute value of the deformation depends on the size of the deformable body.

For example, the Earth must be deformed into an ellipsoid in the field of gravitational radiation, stretched (compressed) perpendicular to the direction of the incoming wave, and the degree of stretching (compression) varies with the frequency of the incoming of gravitational radiation (Figure 2).

As a result, seismic waves are excited by the action of the tidal force $\boldsymbol{F}$ in the body of the Earth's [8]. If $h \approx$ $10^{-21}$ the value of earth surface displacements:

$$
\Delta r \approx \frac{1}{2} h R_{\text {Earth }} \approx 3 \times 10^{-15} \mathrm{~m} .
$$

$h_{\times}$
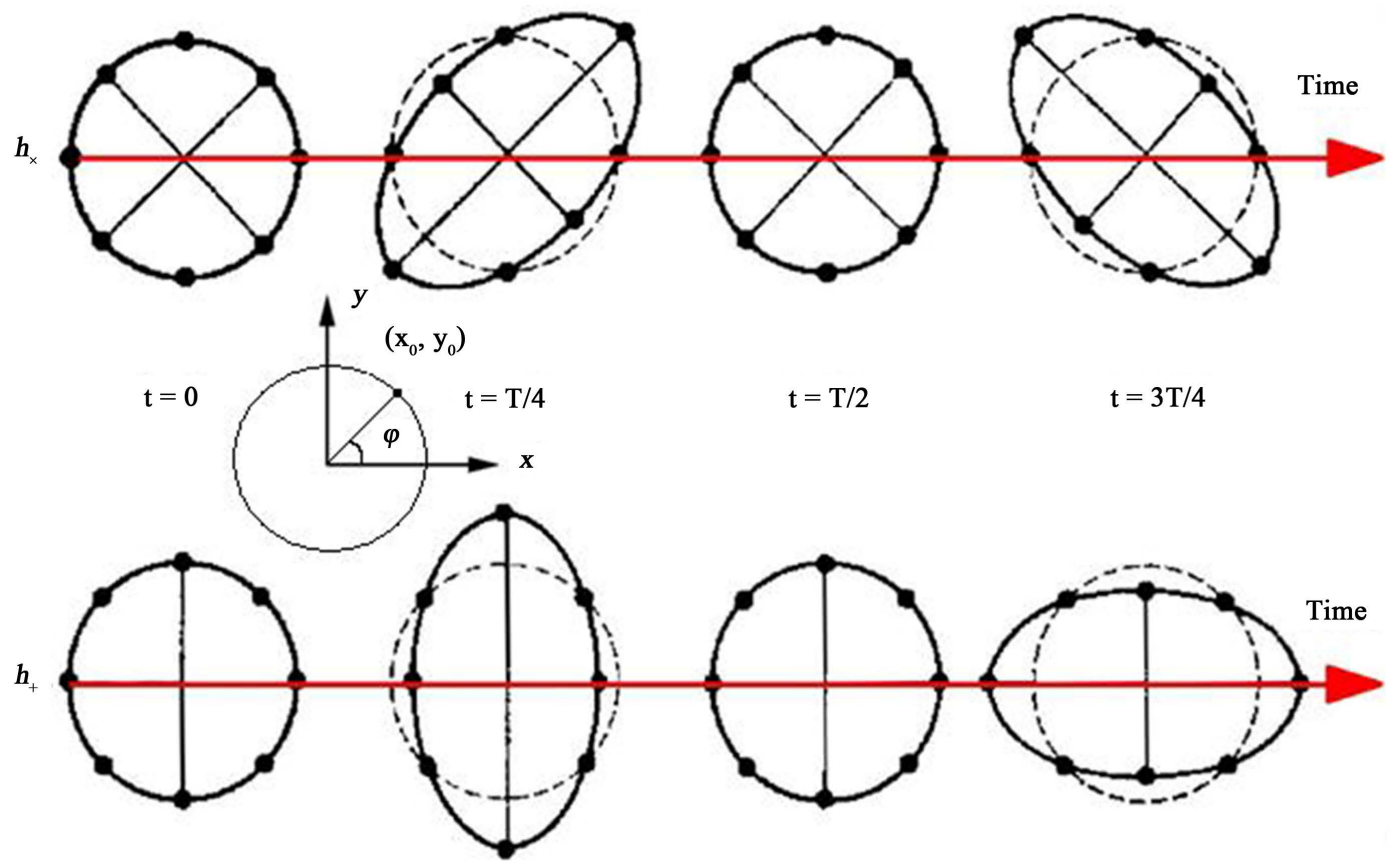

Figure 1. As a gravitational wave passes perpendicular to a ring of test mass it will distort the ring in one of two distinct ways. The “+” and “ $\times$ ” polarisation modes of a GW-wave. The dotted lines (circles) indicate the test particles position in the absence of GW signal. Each step in the graph corresponds to a quarter of the period of the driving GW-wave (solid line). 


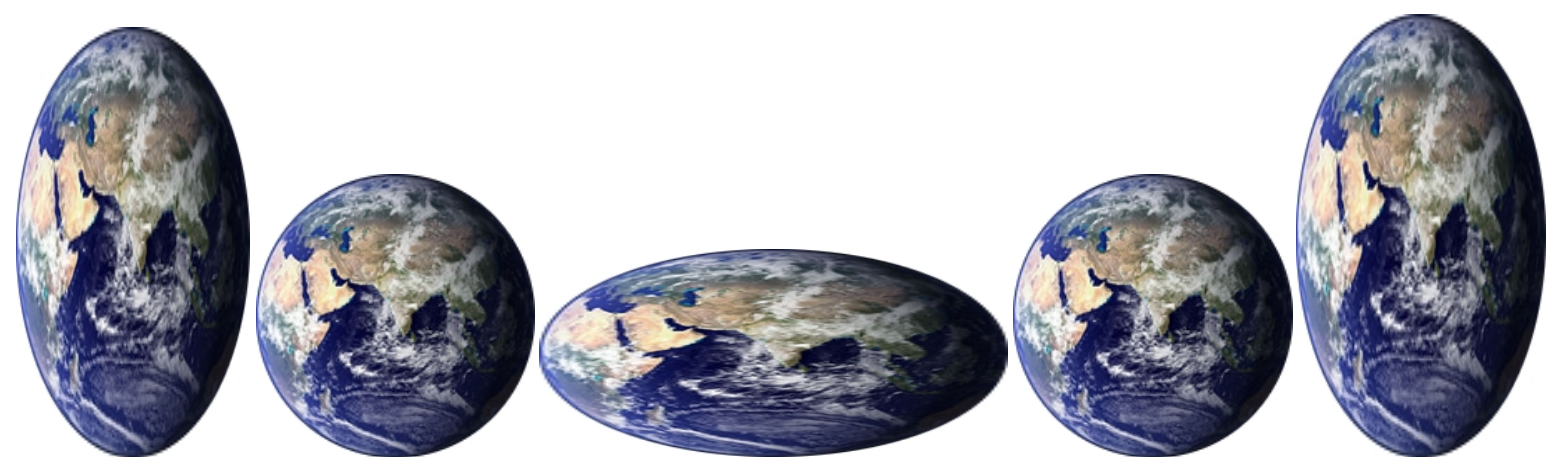

Figure 2. The deformation of the Earth during the passage of a gravitational wave. For illustrative the deformation was increased by 21 order. The wave propagates perpendicular to the plane of the sheet, $h_{+} \neq 0, h_{x}=0$.

Such displacements are registered using seismometers and better use the network of seismometers located on different continents. Such network is a global seismic antenna (GSA).In this paper we consider the variant of GSA, based on 19 seismic stations of the International Monitoring System (IMS) of the Comprehensive Nuclear Test Ban Treaty (CTBT), placed on different continents (Figure 3) [9].

To detect displacements of the Earth's surface, threshold sensitivity of GSA must be:

$$
P_{\mathrm{GSA}} \leq 10^{-15} \mathrm{~m}=1 \text { Fermi. }
$$

Registration of displacements is carried against the background seismic noise and the main restriction on sensitivity of GSA is the natural seismic noise.

\section{Description of Seismic Noises}

Natural seismic fields are generated by natural processes in the Earth's interior, and have different physical nature. The field of seismic noise has two main components: diffuse and coherent [10]-[14].

The diffuse component is generated by a large number of simultaneously acting randomly distributed in space and unrelated sources. This is the result of the spontaneous seismic emission. Corresponding diffuse field accurately described as a homogeneous Gaussian field with a small radius of spatial correlation (not more than 10 $20 \mathrm{~km}$ at frequencies greater than $1 \mathrm{~Hz}$ ). Their spectral density has a "smooth" form without defined peaks at any frequencies [10]-[14].

Coherent component is generated by strong sources of noise, localized in space.

Fields of storm microseisms have clearly coherent nature, center frequency $f \approx 0.2 \mathrm{~Hz}$. Local seismic sources (a distance of less than $100 \mathrm{~km}$ ) cause the appearance of coherent components at frequencies greater than $1 \mathrm{~Hz}$ and they are specific to each station.

Placements of IMS stations are selected so as to minimize the coherent component, and diffuse component would be decisive.

It follows that the seismic noises at the stations practically are not correlated with each other, if the stations are spaced apart at distances of several thousand kilometers.

They do not have common sources of seismic noise.

Selected stations (Figure 3) are the main (primary) stations in the IMS CTBT, and they - one of the quietest stations on the Earth. Seismometers of these stations are installed at a depth 30-100 m in bedrock outcrops, far away from many industrial sources of seismic noise and, as a rule, far from the sea and ocean shores. Such conditions of stations placement provide minimum levels of seismic noise.

Power spectrum of noise at these stations are generally close to or lower than the corresponding curve for a "quiet" conditions, as shown in Figure 4.

The following characteristics of seismic noise at frequencies above $1 \mathrm{~Hz}$ will be considered further [10]-[14]: -normal distribution of seismic noise;

-noises on seismic stations are independent, if stations are spaced apart from one another at distances of several hundred kilometers or more;

-power spectral density $G_{n}(f) \leq 10^{-18}(\mathrm{~m} / \mathrm{sec})^{2} / \mathrm{Hz}$ for total antenna beam of “quiet” seismic arrays; 


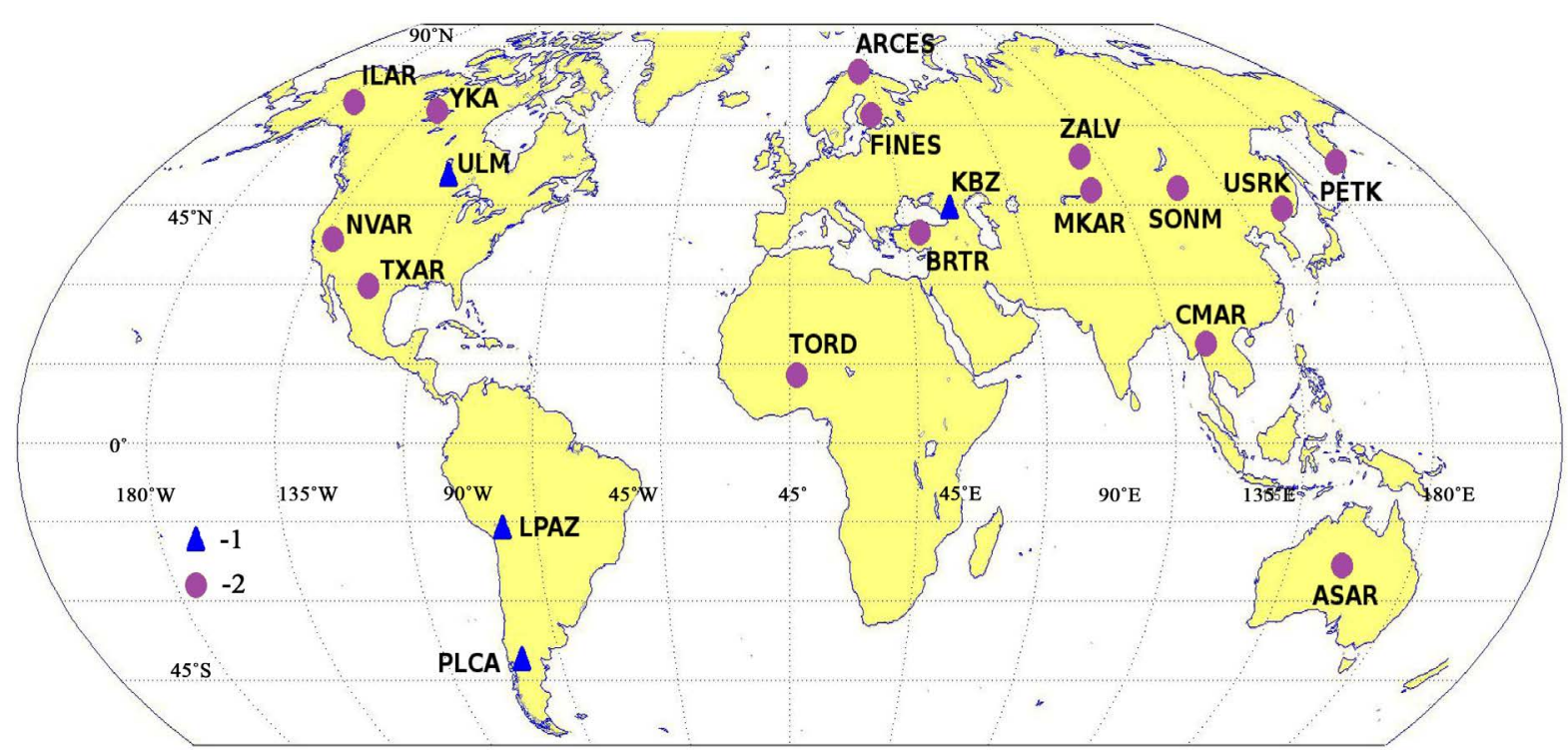

Figure 3. Location of selected stations IMS CTBT [9]. 1-three-component station; 2-seismic array.

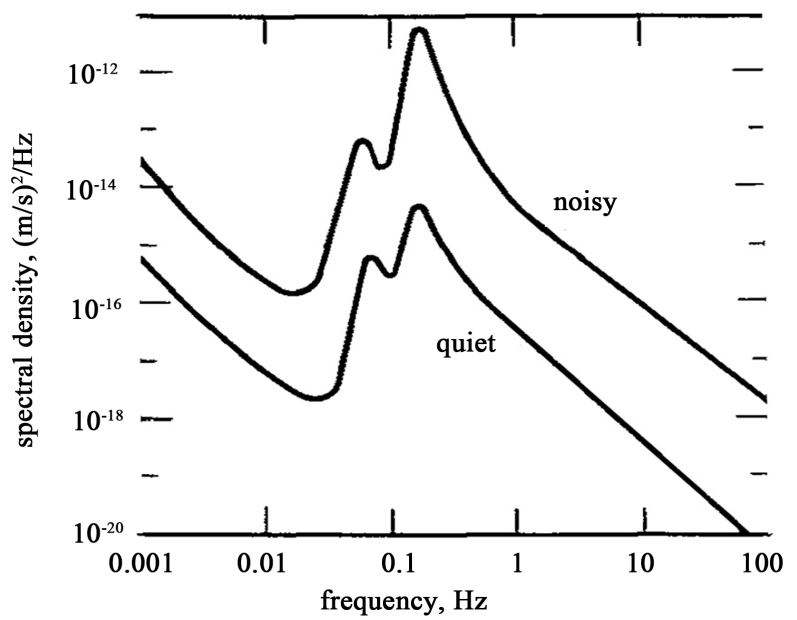

Figure 4. The power density spectrum of seismic noise in "noisy" and "quiet” conditions for a typical station on solid rock (single registration point) [10].

-power spectral density decreases with increasing frequency $G_{n}(f) \approx G_{n}(1) f^{-2}$;

-stationarity interval of seismicnoise $\approx 6-8$ hours in the frequency band less than $0.1 \mathrm{~Hz}$.

15 (fifteen) seismic arrays (SA) are the basis of the seismic network (Figure 3), their aperture of no more than $25 \mathrm{~km}$. The seismic noise dispersion of the total antenna beam decreases by $N_{\text {sensor }}$ times, $N_{\text {sensor }}$-number of SA sensors.

Frequencies more than $1 \mathrm{~Hz}$ allow to achieve the highest sensitivity of GSA and they are preferred for the detection of assumed periodic sources because many periods of pulsars are less $2 \mathrm{sec}\left(T_{\text {rot }}<2 \mathrm{~s}\right.$, rotation frequency $f_{\text {rot }}>0.5 \mathrm{~Hz}$ and $f_{\text {pul }}>1 \mathrm{~Hz}$ ).

\section{Mathematical Model of the Gravitational Signal}

The response of test mass under the action of gravitational waves on the surface of the Earth [15] [16]:

$$
m_{p} \frac{\mathrm{d}^{2} x_{p}^{m}}{\mathrm{~d} t^{2}}=-m_{p} c^{2} R_{m 0 n 0} x_{p}^{n}+F_{p}^{m}, n, m=1,2,3 ;
$$


where $x_{m}^{p}$-coordinates of the mass element;

$F_{p}^{m}$ - external tidal force;

$m_{p}$ - test mass;

$R_{m 0 n 0}$-Riemann tensor.

In the particular case it is possible to convert the Equation (1), for example, if GW-wave falls on seismometer (detector) with an angular frequency $\omega=2 \pi f$ in the direction $\mathbf{z}$, then motion of the mass element of seismometer under the action of GW-wave is described by equations in its own local frame [16] [17]:

$$
\begin{gathered}
\left(\begin{array}{l}
\ddot{x} \\
\ddot{y}
\end{array}\right)=A(t)\left(\begin{array}{l}
x \\
y
\end{array}\right)+F(t), \quad \ddot{z}=0, \\
A(t)=\frac{1}{2}\left(\begin{array}{ll}
\ddot{h_{+}}\left(t-\frac{z}{c}\right) & \ddot{h}_{x}\left(t-\frac{z}{c}\right) \\
\ddot{h_{x}}\left(t-\frac{z}{c}\right) & -\ddot{h}_{+}\left(t-\frac{z}{c}\right)
\end{array}\right), \quad F(t)=\left(\begin{array}{l}
F_{x}(t) \\
F_{y}(t)
\end{array}\right) .
\end{gathered}
$$

If the source is periodic, then the matrix $A(t)$ and the force $F(t)$ are periodic.

Therefore, solutions of the system (Equations (2)) are also periodic functions:

$$
x(t)=x(t+T), y(t)=y(t+T), T=1 / f,
$$

and they can be expanded in a Fourier series:

$$
x(t)=\sum_{n=1}^{\infty} a_{x}(n) \cos (n \cdot 2 \pi f t)+b_{x}(n) \cdot \sin (n \cdot 2 \pi f t), \quad y(t)=\sum_{n=1}^{\infty} a_{y}(n) \cos (n \cdot 2 \pi f t)+b_{y}(n) \cdot \sin (n \cdot 2 \pi f t) .
$$

First approximations of these solutions are harmonic components $(n=1)$ :

$$
x_{1}(t)=a_{x}(1) \cos (2 \pi f t)+b_{x}(1) \cdot \sin (2 \pi f t), \quad y_{1}(t)=a_{y}(1) \cos (2 \pi f t)+b_{y}(1) \cdot \sin (2 \pi f t) .
$$

For an arbitrary direction of propagation of the GW-wave the useful signals are represented as:

$$
\begin{aligned}
& \boldsymbol{S}(\boldsymbol{r}, t)=a_{\text {disp }}(f, \boldsymbol{p}) \cos (2 \pi f(t+\tau)) \boldsymbol{e}_{1}+b_{\text {disp }}(f, \boldsymbol{p}) \sin (2 \pi f(t+\tau)) \boldsymbol{e}_{2}, \\
& \tau=(\boldsymbol{k}, \boldsymbol{p}), \boldsymbol{k}=\boldsymbol{r} / c, \\
& \boldsymbol{S}(\boldsymbol{r}, t)=\left(S_{1}(\boldsymbol{r}, t), S_{2}(\boldsymbol{r}, t), 0\right), \\
& \boldsymbol{S}(\boldsymbol{r}, t) \in L\left(\boldsymbol{e}_{1}, \boldsymbol{e}_{2}\right), \boldsymbol{e}_{1} \perp \boldsymbol{p}, \quad \boldsymbol{e}_{2} \perp \boldsymbol{p}, \\
& S_{1}(\boldsymbol{r}, t)=a_{\text {disp }}(f, \boldsymbol{p}) \cos (2 \pi f(t+\tau)), S_{2}(\boldsymbol{r}, t)=b_{\text {disp }}(f, \boldsymbol{p}) \sin (2 \pi f(t+\tau)),
\end{aligned}
$$

orthonormal basis $\left(\boldsymbol{e}_{1}, \boldsymbol{e}_{2}, \boldsymbol{p}\right)$;

$\boldsymbol{r}=\boldsymbol{r}(\varphi, \lambda)=R_{E}(\cos \varphi \cos \lambda, \cos \varphi \sin \lambda, \sin \varphi)$-vector from the center of the Earth to the point on the surface with coordinates $(\varphi, \lambda)$; Earth radius $R_{E}=6,371,000 \mathrm{~m} ; \boldsymbol{p}=\left(\cos \varphi_{p} \cos \lambda_{p}, \cos \varphi_{p} \sin \lambda_{p}, \sin \varphi_{p}\right)$-vector directed to the point of the celestial sphere $\left(\varphi_{p}, \lambda_{p}\right)$ (second equatorial coordinate system).

Signal (Equation (3)) - the ellipse in the plane perpendicular to the direction $\boldsymbol{p}, a_{\text {disp }}(f, \boldsymbol{p})$ and $b_{\text {disp }}(f, \boldsymbol{p})$ — semiaxes of the ellipse.

Canonical equations of ellipses, describing motion of the mass element in the coordinate system of the detector, are given in many works, in particular [17] [18].

Ellipticity of solutions of Equations (2) is a property of stable solutions of linear differential equations with periodic coefficients.

The particular case $x_{0}=r_{0} \cos (\varphi), y_{0}=r_{0} \sin (\varphi), z_{0}=0$ (Figure 1 ).

If $\mathrm{GW}$-wave falls in the direction $\mathbf{z}$, then motion near $\left(x_{0}, y_{0}\right)$ is described by equations: 


$$
\begin{aligned}
& x(t)=x_{0}+\frac{1}{2}\left(h_{+}(t) x_{0}+h_{\times}(t) y_{0}\right), t>0 \\
& y(t)=y_{0}+\frac{1}{2}\left(h_{\times}(t) x_{0}-h_{+}(t) y_{0}\right), \\
& z(t)=z_{0}
\end{aligned}
$$

a) $h_{x}=0$.

$$
\begin{gathered}
x(t)=r_{0} \cos (\phi)\left(1+\frac{1}{2} h_{+}(t)\right), y(t)=r_{0} \sin (\phi)\left(1-\frac{1}{2} h_{+}(t)\right), z_{0}=0 \\
\frac{x(t)}{r_{0} \cos (\varphi)}+\frac{y(t)}{r_{0} \sin (\varphi)}=2
\end{gathered}
$$

b) $h_{+}=0$.

$$
\frac{x(t)}{r_{0} \cos \left(\phi-\frac{\pi}{4}\right)}+\frac{y(t)}{r_{0} \sin \left(\phi-\frac{\pi}{4}\right)}=2
$$

Point of circle $\left(x_{0}, y_{0}\right)$ moves in a straight line for cases a) and b), the total motion of point $\left(x_{0}, y_{0}\right)$ is elliptical, if $h_{+} \neq 0$ and $h_{x} \neq 0$.

Motion of point $\left(x_{0}, y_{0}\right) \quad\left(r_{0}=R_{\text {Earth }}\right)$ on the Earth's surface is same elliptical, and it's independent of the Earth rocks.

General Relativity is the geometrical theory, the principle equivalence of inertial and gravitational mass is observed. The bars of equal length are stretched identically and it does not depend on the material from which they are made.

Equation (1) describes the primary effect of GW-wave on matter.

\section{Detector of Gravitational Signals}

Registered seismic process can be represented as:

$$
\boldsymbol{x}(\boldsymbol{r}, t)=\boldsymbol{s}(\boldsymbol{r}, t) \cdot \delta+\boldsymbol{n}(\boldsymbol{r}, t),
$$

where

$\boldsymbol{s}(\boldsymbol{r}, t)$ — detectable signal (Equation (3)); $\boldsymbol{n}(\boldsymbol{r}, t)$ — seismic noise;

$\delta=0$ or 1 -signal is absent or present, respectively.

Because the third component of the signal is zero, it is sufficient to consider the projection of seismic processes on the plane $L\left(\boldsymbol{e}_{1}, \boldsymbol{e}_{2}\right)$ for detection:

$$
\begin{aligned}
& y(\boldsymbol{r}, t)=\boldsymbol{x}_{\mathrm{pr}}(\boldsymbol{r}, t), \\
& \boldsymbol{y}(\boldsymbol{r}, t)=\boldsymbol{s}_{\mathrm{pr}}(\boldsymbol{r}, t) \cdot \delta+\boldsymbol{n}_{\mathrm{pr}}(\boldsymbol{r}, t), \\
& \boldsymbol{s}_{\mathrm{pr}}(\boldsymbol{r}, t)=\left(S_{1}(\boldsymbol{r}, t), S_{2}(\boldsymbol{r}, t)\right), \boldsymbol{s}_{\mathrm{pr}}(\boldsymbol{r}, t)=\boldsymbol{s}(\boldsymbol{r}, t),
\end{aligned}
$$

where $\boldsymbol{y}(\boldsymbol{r}, t)\left(\boldsymbol{S}_{\mathrm{pr}}(\boldsymbol{r}, t), \boldsymbol{n}_{\mathrm{pr}}(\boldsymbol{r}, t)\right)$-projection of $\boldsymbol{x}(\boldsymbol{r}, t)(\boldsymbol{S}(\boldsymbol{r}, t), \boldsymbol{n}(\boldsymbol{r}, t))$ on the $L\left(\boldsymbol{e}_{1}, \boldsymbol{e}_{2}\right)$, respectively.

Registered data are represented as the vector of observations:

$$
\vec{y}(m)=\left(\boldsymbol{y}_{1}(m), \cdots, \boldsymbol{y}_{i}(m), \cdots, \boldsymbol{y}_{N_{s t}}(m)\right)^{\mathrm{T}},
$$

where

$$
\begin{aligned}
& \boldsymbol{y}_{i}(m)=\boldsymbol{s}_{\mathrm{pr}}\left(\boldsymbol{r}_{i}, m \Delta t\right)+\boldsymbol{n}_{\mathrm{pr}}\left(\boldsymbol{r}_{i}, m \Delta t\right), \\
& \mathrm{s}_{\mathrm{pr}}\left(\boldsymbol{r}_{i}, m \Delta t\right)=\mathrm{s}_{i \mathrm{pr}}(t), \boldsymbol{n}_{\mathrm{pr}}\left(\boldsymbol{r}_{i}, m \Delta t\right)=\boldsymbol{n}_{i \mathrm{pr}}(t), t=m \Delta t,
\end{aligned}
$$




$$
1 \leq m \leq N, 1 \leq i \leq N_{\text {st }} ;
$$

$\boldsymbol{r}_{i}$ - vector from the center of the Earth to the point of placing of the $i$-th station,

$N_{s t}$-number of stations seismic network; $N$-size of the sample data;

$\Delta t$ - sampling interval time, $F_{d}=1 / \Delta t-$ sampling frequency.

Detection of gravitational signal is based on the selection of one from two alternative hypotheses:

$\boldsymbol{H}_{\mathbf{0}}: \quad \boldsymbol{y}_{i}(m)=\boldsymbol{n}_{\mathrm{pr}}\left(\boldsymbol{r}_{i}, m \Delta t\right), 1 \leq i \leq N_{\mathrm{st}}-\mathrm{GW}$-wave signal is absent;

$\boldsymbol{H}_{\mathbf{1}}: \boldsymbol{y}_{i}(m)=\boldsymbol{s}_{\mathrm{pr}}\left(\boldsymbol{r}_{i}, m \Delta t\right)+\boldsymbol{n}_{\mathrm{pr}}\left(\boldsymbol{r}_{i}, m \Delta t\right), 1 \leq i \leq N_{\mathrm{st}}$-GW-wave signal is present.

Optimal detection of signals (Equation (3)) is based on the evaluation of log-likelihood ratio:

$$
L(\vec{y} / s)=\ln \frac{P(\vec{y} / s)}{P(\vec{y} / n)},
$$

where $\vec{y}=\left(\boldsymbol{y}_{1}(t), \cdots, \boldsymbol{y}_{i}(t), \cdots, \boldsymbol{y}_{N_{s t}}(t)\right)$;

$\boldsymbol{y}_{i}(t)$ - projection of seismic process (i-th station);

$P(\vec{y} / s)(P(\vec{y} / n))$ — joint distribution density $\vec{y}$, if signal is present or absent, respectively.

Since seismic noises are independent for different stations, then:

$$
L(\vec{y} / s)=\ln \prod_{i=1}^{N_{s t}} \frac{P\left(\vec{y}_{i} / s\right)}{P\left(\vec{y}_{i} / n\right)}=\sum_{i=1}^{N_{s t}} \ln \frac{P\left(\vec{y}_{i} / s\right)}{P\left(\vec{y}_{i} / n\right)},
$$

where $P\left(\vec{y}_{i} / s\right)\left(P\left(\vec{y}_{i} / n\right)\right)$ - distribution density $y_{i}(t)$, if signal is present or absent, respectively.

Such representation greatly simplifies the evaluation of log-likelihood ratio, and allows using the processing methods of seismic array data.

The formation of total antenna beam (beam forming) is one of the basic methods of seismic data processing:

$y_{\text {beam }}(t)=\frac{1}{N_{\text {st }}} \sum_{i=1}^{N_{s t}} y_{i}\left(t-\tau_{i}\right)$,

where $\tau_{i}$-delays of arrival of the wave at different stations.

Result of the beam forming is elliptical signal, if elliptical signals are at stations of GSA.

The optimal functional of detection of a priori unknown signal is represented as follows:

$$
L_{m}(\vec{y})=\max _{\theta} L(\vec{y} / \theta)
$$

where $\theta=\{a(f, \boldsymbol{p}), b(f, \boldsymbol{p}), \boldsymbol{p}\}$ — set of signal parameters (Equation (3)) [19].

After the simplification of this expression can be shown that the optimal functional of detection evaluates the energy of gravitational radiation coming from an arbitrary point on the celestial sphere $\left(\varphi_{p}, \lambda_{p}\right)$ at frequency $f$ and determines the direction of maximum energy:

$$
\begin{gathered}
L_{m}(\vec{y})=\max _{\boldsymbol{p}} E(f, \boldsymbol{p}), \\
E(f, \boldsymbol{p})=a_{v}(f, \boldsymbol{p})^{2}+b_{v}(f, \boldsymbol{p})^{2}, \\
a_{v}(f, \boldsymbol{p})=\omega \cdot a_{\text {disp }}(f, \boldsymbol{p}), b_{v}(f, \boldsymbol{p})=\omega \cdot b_{\text {disp }}(f, \boldsymbol{p}) .
\end{gathered}
$$

Expression (Equation (5)) is an estimation of energy of the elliptically polarized seismic process (EPSP), $\left(a_{v}(f, \boldsymbol{p})\right.$ and $\left.b_{v}(f, \boldsymbol{p})\right)$-semiaxes of the ellipseby velocity.

Estimation of energy is carried out on the time fragment $\left[t_{0}, t_{0}+T\right]$, whereby $E(f, \boldsymbol{p})$ is a function of the time $t_{0}$ and duration $T$.

Next, we consider estimation $E(f, \boldsymbol{p})$ on the 4-hour fragments ( $T=4$ hours).

To estimate the energy 32 directions (beams) $\boldsymbol{p}$ were chosen:

$$
\phi_{p}=-60^{\circ}+\left(k_{\phi}-1\right) d \phi, \lambda_{p}=\left(k_{\lambda}-1\right) d \lambda, k_{\phi}=1 \div 4, k_{\lambda}=1 \div 8, d \phi=45^{\circ}, d \lambda=22.5^{\circ}
$$


and you must find the $\boldsymbol{p}$, for which the maximum value of $E(f, \boldsymbol{p})$ is achieved.

\section{Sources of Periodic Gravitational Radiation}

Sources of harmonic signals, naturally, are associated with pulsars. The radio emission signals (RES) of pulsar PSR $1919+21$ are shown in Figure 5. The stability of the pulsar period follows from it, but this property does not apply to the form of these signals. The spectrum of temporary fragment (Figure 5) is shown in Figure 6.

The visible frequency (Figure 5) of these signals: $f_{\text {vis }}=1 / P=1 / 1.33730113=0.747 \mathrm{~Hz}$.

The center frequency $f_{\text {cent }}=0.77 \mathrm{~Hz}$ significantly differs from the visible frequency, shift ( $\Delta f \approx 0.023 \mathrm{~Hz}$ ) is $3 \%$ of the center frequency: $\frac{\left|f_{\text {vis }}-f_{\text {cent }}\right|}{f_{\text {cent }}} \approx 3 \%$.

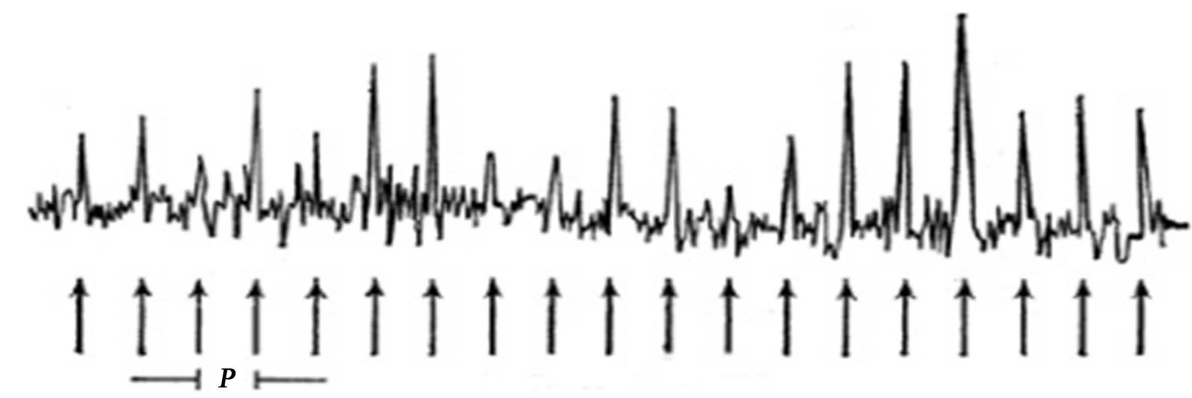

Figure 5. Signals of radio emission PSR $1919+21$ at a frequency of $72.7 \mathrm{MHz}$ [20]. Pulsar period ( $P$ was equal to $1.33730113 \mathrm{~s}$ ) at the time of its opening.

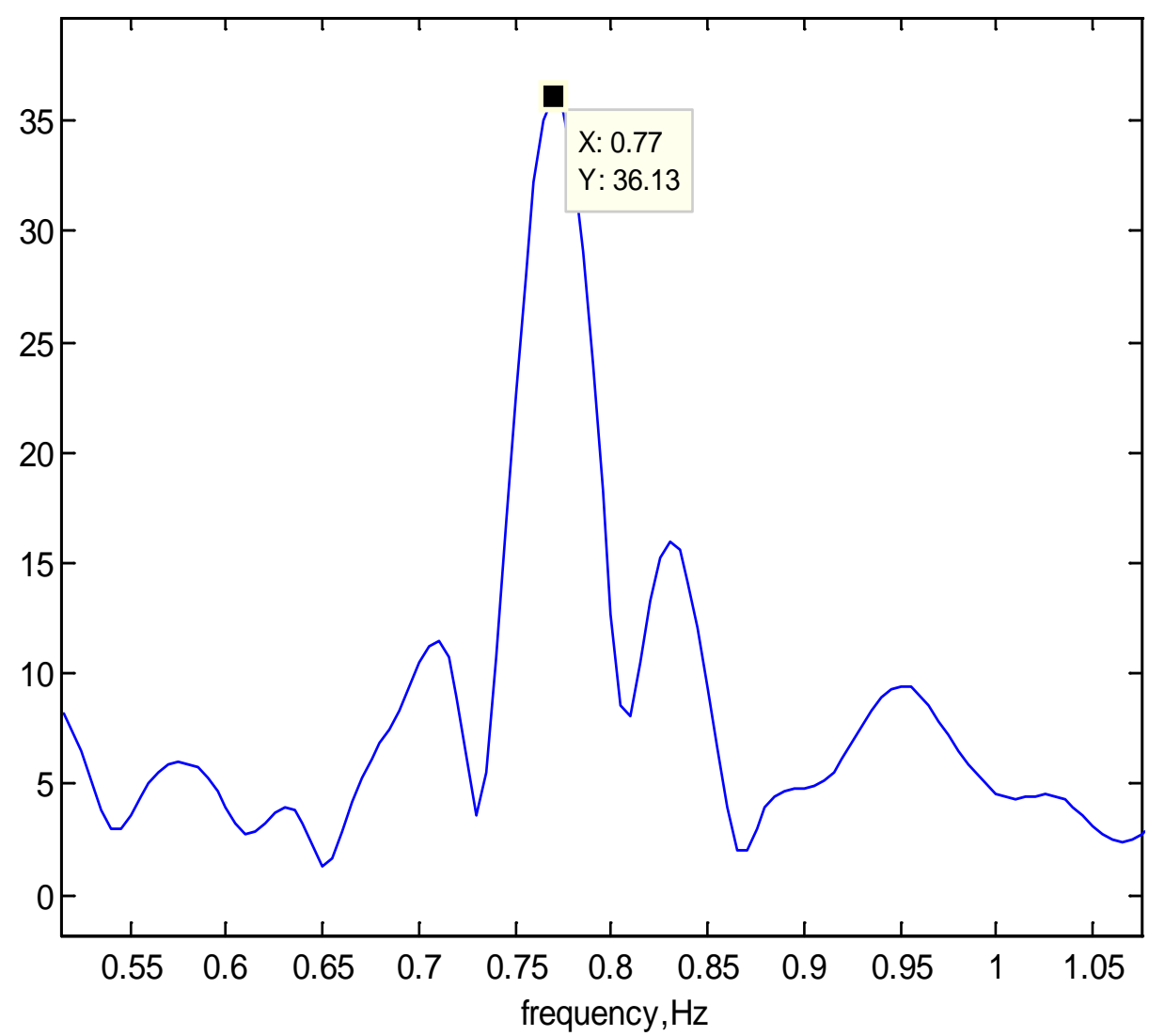

Figure 6. The spectrum of radio emission signals PSR $1919+21$. 
Instability of RES forms is observed for many pulsars [21] [22], so we can assume, that property is observed for these signals:

Center frequency can differ a few percent from the visible frequency, specified in the catalogs of pulsars.

Pulse repetition periods of the observed pulsars lie in the range of $\approx 1.6 \mathrm{~ms}$ to $\approx 4.3 \mathrm{~s}$. The emission of pulsars is generally strongly polarized, the degree of polarization of the radio emission is close to $100 \%$, almost circular polarization radio emission is observed for some pulsars [22].

Gravitational wave radiation (GWR) of the pulsar is determined by the quadrupole moment of the source. Given a priori ignorance of the quadrupole moment, we can assume that the complexity of gravitational radiation is similar to the complexity of pulsar radio emission. Consequently, the spectrum of GWR is the convolution of the spectra of the modulating function and aoriginal signal and spectrum of GWR expands and "floats" near the center frequency.

Most of the energy of the signal (Figure 6) is concentrated in the band $\Delta f \approx 0.03 \mathrm{~Hz}$.

The detection needs to produce in the frequency bands $\left(\Delta f_{\text {det }}<0.03 \mathrm{~Hz}\right)$ to provide a minimum loss the noise immunity.

\section{Theoretical Estimation of GSA Sensitivity}

Detection of useful harmonic signals is carried out in bands having a width $\Delta f \approx 0.01 \mathrm{~Hz}$.

Evaluation of seismic noise dispersion in the frequency band $[f-\Delta f / 2, f+\Delta f / 2]$ :

$$
D_{i}=\sigma_{i}^{2}=\int_{f-\frac{\Delta f}{2}}^{f+\frac{\Delta f}{2}} G_{n_{i}}(u) \mathrm{d} u
$$

where $G_{n_{i}}(f)$-the power density spectrum of the seismic noise on the $i$-th station.

Using this equation for total antenna beam in each array, the evaluation of seismic noise dispersion in the frequency band $[f-\Delta f / 2, f+\Delta f / 2]$ is equal to:

$$
\begin{gathered}
D_{n}(f)=\sigma_{n}^{2}(f) \approx G_{n}(f) \cdot \Delta f \leq 10^{-18} \cdot \Delta f \cdot f^{-2}(\mathrm{~m} / \mathrm{s})^{2}, \\
D_{n}(f) \leq 10^{-20} f^{-2}(\mathrm{~m} / \mathrm{s})^{2} \quad \text { if } \Delta f=0.01 \mathrm{~Hz} .
\end{gathered}
$$

If the signal $s(t)=A \cos (\omega t+\varphi)$, for evaluation of its amplitude are calculated values:

$$
A_{\text {cos }}=\frac{1}{N \cdot N_{\text {st }}} \sum_{m=1}^{N} \sum_{i=1}^{N_{s t}} y_{i}(m) \cos \left(\omega\left(m \Delta t-\tau_{i}\right)\right), \quad A_{\text {sin }}=\frac{1}{N \cdot N_{\text {st }}} \sum_{m=1}^{N} \sum_{i=1}^{N_{s t}} y_{i}(m) \sin \left(\omega\left(m \Delta t-\tau_{i}\right)\right)
$$

where $\tau_{i}$-corresponding of signal arrival delays, and evaluation of the amplitude is carried out according to the formula: $\hat{A}=2 \sqrt{A_{\text {sin }}^{2}+A_{\text {cos }}^{2}}$.

Dispersion of the noise is decreased by $V$ times due to the processing of seismic data for a long time:

$$
V \approx N_{\text {st }} \cdot N_{\text {uncorr. }} \approx N_{\text {st }} \cdot T_{\text {an }} \cdot 2 \cdot \Delta f,
$$

where $N_{\mathrm{st}}$-number of stations of GSA;

$N_{\text {uncorr }}$-number of uncorrelated time values;

$T_{\mathrm{an}}$-duration of analysis fragment;

$\Delta f$-band of frequency analysis.

For example, dispersion of evaluation of amplitude:

$$
\begin{gathered}
D_{\Sigma}(f)=D\left(A_{\text {cos }}\right)+D\left(A_{\text {sin }}\right)=\sigma_{\Sigma}^{2}(f) \leq \sigma_{n}^{2}(f)(1 / V)=(1 / V) \cdot 10^{-20} \cdot f^{-2}=10^{-24} \cdot f^{-2}(\mathrm{~m} / \mathrm{s})^{2}, \\
D_{\Sigma}(f) \leq 10^{-24} \times 6^{-2} \approx 2.7 \times 10^{-26}(\mathrm{~m} / \mathrm{s})^{2}, \\
\sigma_{\Sigma v}(f) \leq 1.6 \times 10^{-13}(\mathrm{~m} / \mathrm{s}), \text { if } T=8 \times 3600 \mathrm{~s}, \Delta f=0.01 \mathrm{~Hz}, \quad f=6.023 \mathrm{~Hz} .
\end{gathered}
$$


The amplitudes of the velocity and displacement are related:

$$
A_{\text {displ }}=A_{v} /(2 \pi \cdot f)
$$

Corresponding standard deviation of the noise amplitude (displacement):

$$
\sigma_{\Sigma \text { displ }}=\sigma_{\Sigma v} /(2 \pi \cdot f) \leq 4.4 \times 10^{-15} \mathrm{~m} \text { if } f \approx 6 \mathrm{~Hz} \text {. }
$$

This is an upper rough estimation of GSA sensitivity threshold near $6 \mathrm{~Hz}$, and it characterizes the detection capabilities of GSA. This estimate is obtained theoretically using known characteristics and properties of the seismic noises.

More accurate estimate can be obtained if one considers that the signal is not only the harmonic, but also has an elliptical polarization.

Optimum processing functional (Equation (4)) tuned to detect a harmonic elliptically polarized signals for different arbitrary sources.

The energy of noise can be represented as the sum of the energies of the polarized and unpolarized components, elliptical polarization of noise represents a strict condition, and it leads to a significant reduction of a level of the elliptical polarized component:

$$
\sigma_{\text {¿displ }} \gg \sigma_{\text {ellips polar }}=\sigma_{\text {EPSP }} .
$$

\section{The Results of Processing of GSA Data on $\mathbf{9 0 - H o u r ~ I n t e r v a l ~}$}

Processing of GSA data was performed on a time interval $00^{00} 27.02 .2009-18^{00} 2.03 .2009$.

By averaging the values of the energies at a frequency $f$ by space, we estimate the energy, received by the antenna at a given frequency $E_{\text {aver }}\left(f, t_{0}\right)=\sum_{\boldsymbol{p}} E(f, \boldsymbol{p})$ on the fragment $\left[t_{0}, t_{0}+T\right]$.

Estimating the energies on time fragments (shift $=1$ hour), you can get a diagram of spectral-time analysis, $0 \leq t_{0} \leq 90$ hours, frequency step $d f=40 / 4096 \mathrm{~Hz}$ (Figure 7). The energies are changed in the range from $1 \times$ $10^{-27}(\mathrm{~m} / \mathrm{s})^{2}$ to $35 \times 10^{-27}(\mathrm{~m} / \mathrm{s})^{2}$.

Analyzing Figure 7, one can observe a strong signal for 90 hours ( $\approx 4$ days) in the band from 6.00 to $6.04 \mathrm{~Hz}$ with a center frequency $f_{0}=6.023 \mathrm{~Hz}$.

Estimating the significance level (the error of the $\mathbf{1}^{\text {st }} \mathbf{k i n d}$ ), it is possible to confirm the hypothesis that the fact of energy ejection at the frequency $f_{0}=6.023 \mathrm{~Hz}$ is not random.

It is also possible to evaluate the average energies (Figure 8) during 90 hours:

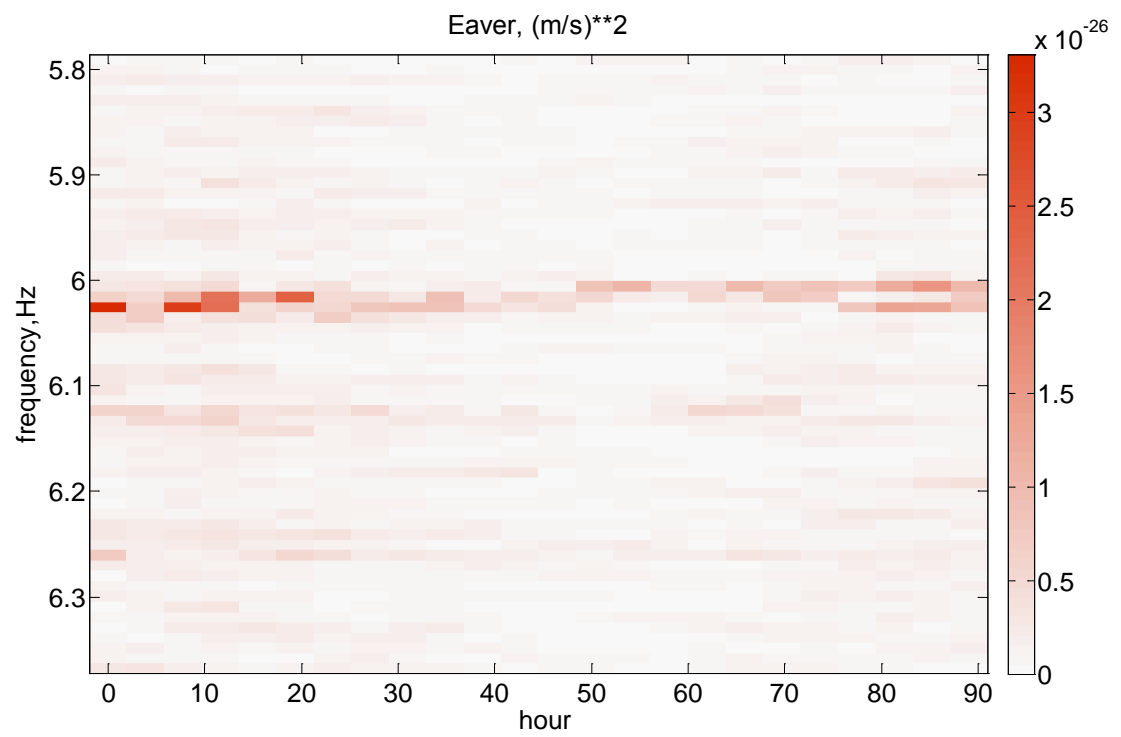

Figure 7. The average energies of EPSP Eaver $\left(f, t_{0}\right)$ for $00^{00} 27.02 .2009-18^{00}$ 2.03.2009. The frequency band $[5.8 \div 6.4 \mathrm{~Hz}]$. 


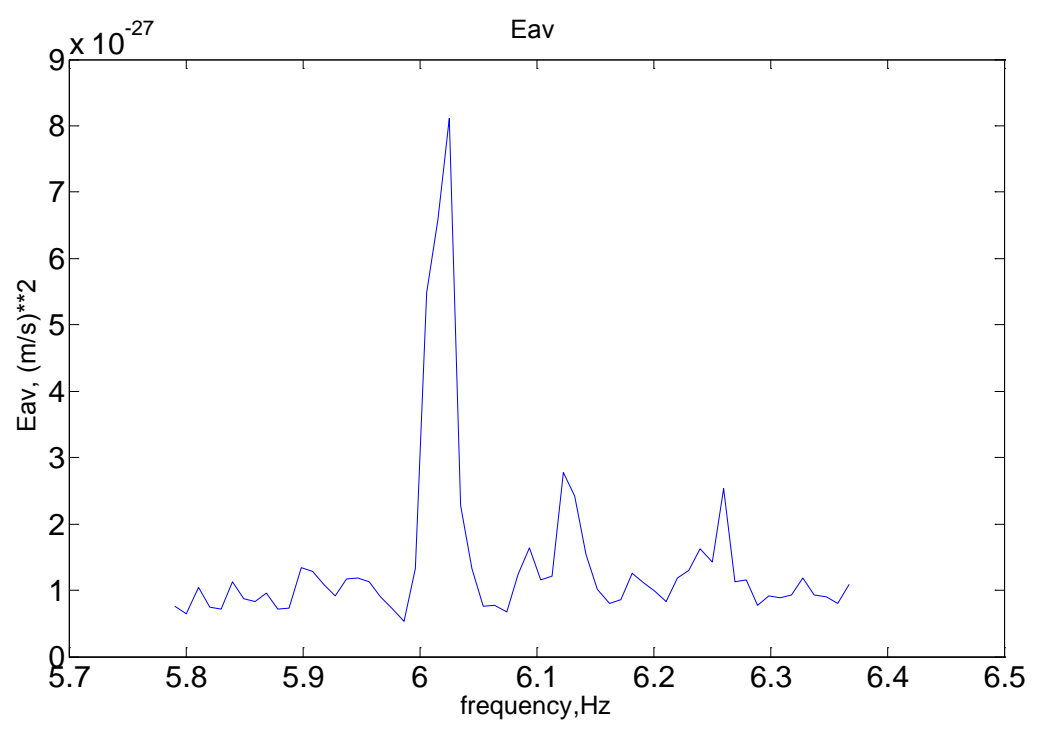

Figure 8 . The average energies of EPSP by time and space for 90 hours.

$$
E_{a v}(f)=\frac{1}{90} \sum_{t_{0}=0}^{89} E_{\text {aver }}\left(f, t_{0}\right),
$$

$E_{a v}(f)$ - the average energies of EPSP by time and space.

\section{Confidence Probability of Detection}

Spectrum $E_{a v}(f)$ (Figure 8 ) is the result of averaging 90 energy spectra (including strictly $90 / 4 \approx 22$ independent spectra and equal to the number of spectra on disjoint time intervals), and therefore we can assume that

$E_{a v}(f)$ practically has a normal distribution at each frequency.

Using the values of $E_{a v}(f)$ (Figure 8) at the frequencies of the left and right of the band [5.99 $\left.\div 6.05 \mathrm{~Hz}\right]$, we can estimate the mathematical value of $M\left(E_{a v}\right) \approx 1.2 \times 10^{-27}(\mathrm{~m} / \mathrm{s})^{2}$, standard deviation $\sigma_{E_{a v}}$ satisfies:

$$
0.2(\mathrm{~m} / \mathrm{s})^{2} \leq \sigma_{E_{a v}} \cdot 10^{27} \leq 0.49(\mathrm{~m} / \mathrm{s})^{2} .
$$

The amplitude of the ejection of

$$
A\left(f_{0}\right) \approx 8 \times 10^{-27}(\mathrm{~m} / \mathrm{s})^{2} \text { and } S N R=\Delta\left(f_{0}\right) / \sigma_{\text {Eav }} \geq 12.8
$$

where $\Delta\left(f_{0}\right)=A\left(f_{0}\right)-M\left(E_{a v}\right)$.

The error of the $1^{\text {st }}$ kind:

$$
\alpha \leq P(\xi>12.8),
$$

where $\xi=N(0,1)$ —normally distributed random variable.

The value $\alpha$ is close to zero,the confidence probability of detection $P_{\text {conf }}=1-\alpha$ is close to 1 .

The minimum values $E_{a v}(f)$ (Figure 8) determine threshold of GSA sensitivity at frequencies close to 6 $\mathrm{Hz}$.

Achieved threshold of GSA sensitivity at frequencies close to $6 \mathrm{~Hz}$ :

$$
\begin{gathered}
D_{\sum E P S P} \approx 10^{-27}(\mathrm{~m} / \mathrm{s})^{2} \text { (energy) } \\
V_{\sum E P S P} \approx 0.3 \times 10^{-13} \mathrm{~m} / \mathrm{s} \text { (velocity); } \\
a_{\sum E P S P} \approx 0.8 \times 10^{-15} \mathrm{~m} \text { (displacement) } .
\end{gathered}
$$


This sensitivity of GSA allows detecting deformation of the Earth (Figure 2).

The average value of energy of the detected signal at the frequency $f=6.023 \mathrm{~Hz}$ for 90 hours is equal to $E_{a v}(6.023) \approx 0.8 \times 10^{-26}(\mathrm{~m} / \mathrm{s})^{2}$ (Figure 8 ), corresponding velocity $\approx 0.9 \times 10^{-13} \mathrm{~m} / \mathrm{s}$ and corresponding displacement:

$$
A_{\text {sdet }} \approx 0.9 \times 10^{-13} \mathrm{~m} / \mathrm{s} /(2 \pi \cdot f)=2.5 \times 10^{-15} \mathrm{~m} .
$$

Thus, it is experimentally confirmed (Equation $(11)$ ), that relative displacements $\left(\approx 10^{-15} \mathrm{~m}\right)$ of different points of the Earth are detected using the optimal processing data of global seismic antenna.

The semimajor axis is more than twice the semiminor axis for the corresponding detected signals [19]:

$$
|a(f, \boldsymbol{p})|>2|b(f, \boldsymbol{p})| \text { or }|b(f, \boldsymbol{p})|>2|a(f, \boldsymbol{p})|
$$

and therefore the eccentricity $\varepsilon(f, \boldsymbol{p})>0.866$.

These motions are an objective reality, because a false alarm is practically zero and confidence probability $P_{\text {conf }} \approx 1$.

Therefore search of possible sources of the corresponding signals is performed further in this paper.

It should be noted that the detection of GW-signals (Equation (4)) is based on the phasing of the antenna at the source beyond Earth assuming that the propagation speed of the wave equal to the speed of light, and that signals were detected during a prolonged period of time (90 hours).

Also, the local maxima are seen at the frequencies $f=6.12 \mathrm{~Hz}$ and $f=6.26 \mathrm{~Hz}$ with a smaller SNR, than at the frequency $f=6.023 \mathrm{~Hz}$ (Figure 8).

Errors of $\mathbf{1}^{\text {st }}$ kind $\alpha_{6.12}=P(\xi>6.5), \alpha_{6.26}=P(\xi>7)$ are small and, by analogy, you can also find appropriate sources of gravitational-wave radiation.

\section{Characteristics of the Detected Signals}

Local deformation caused by signal (Equation (3)) can be estimated:

$$
D_{\text {loc }}=\left|\frac{\partial \boldsymbol{S}(\boldsymbol{r}, t)}{\partial r}\right| \approx \frac{\omega}{c} \sqrt{a_{\text {disp }}(f, \boldsymbol{p})^{2}+b_{\text {disp }}(f, \boldsymbol{p})^{2}}, \omega=2 \pi f .
$$

Estimation of $D_{\text {loc }}, h_{0}$ can be obtained (if $f=f_{0}$ ), using Equation (5):

$$
D_{\text {loc }} \approx \frac{\sqrt{E\left(f_{0}, \boldsymbol{p}\right)}}{c}, h_{0}=2 \cdot D_{\text {loc }}
$$

and local deformation (strain) for $f_{0}=6.023 \mathrm{~Hz}$ can be estimated:

$$
D_{\text {loc }}=\left|\frac{\partial \boldsymbol{S}(r, t)}{\partial r}\right| \approx \frac{\omega}{c} \sqrt{a(f, \boldsymbol{p})^{2}+b(f, \boldsymbol{p})^{2}}=\frac{2.5 \times 10^{-15} \times 2 \pi \times 6.023}{3 \times 10^{8}} \approx 3.15 \times 10^{-22} .
$$

The relative change in the distance between stations is

$$
\Delta L_{i, j} \approx \max \left|\boldsymbol{s}\left(\boldsymbol{r}_{i}, t\right)-\boldsymbol{s}\left(\boldsymbol{r}_{j}, t\right)\right|, i, j \text {-station numbers. }
$$

The phase shift between the stations is unknown, in the worst case, $\Delta L_{i, j}$ is less than double amplitude of the detected signal.

Deformation space can be estimated by the change in the distance between any stations on the network, for example between stations ZALV and TXAR (Figure 3):

$$
D_{g l}=\frac{\Delta L}{L_{Z A L V-T X A R}} \leq \frac{2 \times 2.5 \times 10^{-15}}{9498 \times 10^{3}} \approx 5.26 \times 10^{-22},
$$

where $L_{\text {ZALV-TXAR }}=9498 \mathrm{~km}$.

From a comparison of the two estimates of deformation $D_{g l}, D_{l o c}$, it follows, that the velocity of gravitational waves and the speed of light have an equal order. 
Equality of velocity of detected waves and the speed of light is not used in the evaluation $D_{g l}$ and therefore it correct to use to estimate the velocity of propagation of the detected waves and you can use for this the analogue of the formula from the article [23]:

$$
\begin{gathered}
v_{\text {grav }} \approx s_{v} / D_{\text {gl }}, \\
v_{\text {grav }} \geq 0.9 \times 10^{-13} / 5.26 \times 10^{-22} \approx 1.7 \times 10^{8} \mathrm{~m} / \mathrm{s} .
\end{gathered}
$$

\section{The Energy Characteristics of GW-Wave}

The energy of a full rotation of the pulsar:

$$
E_{p u l}=\frac{J \cdot \omega_{p u l}^{2}}{8}, \omega_{r}=2 \pi f_{r}, \omega_{p u l}=2 \pi f_{p u l}, f_{p u l}=2 f_{r}
$$

where $J$-moment of inertia about the axis of rotation;

$f_{r}$-rotation frequency of pulsar;

$f_{\text {pul }}$-frequency of GW-radiation. [24]:

The maximum of energy, emitted per unit of frequency interval, in the form of gravitational radiation is equal

$$
\frac{\mathrm{d} E}{\mathrm{~d} \omega}=\frac{J \omega_{\text {pul }}}{4} .
$$

The spectral energy density of the gravitational-wave radiation (upper bound) at a distance $r_{\text {pul }}$ [24]:

$$
\Phi_{\max }\left(\omega_{p u l}, r_{p u l}\right)=\frac{\Delta E}{\Delta \omega \cdot 4 \pi \cdot r_{p u l}^{2}}=\frac{J \omega_{p u l}}{4 \cdot 4 \pi \cdot r_{p u l}^{2}} .
$$

Such estimates (Equation (15)) are obtained, assuming isotropy of radiation of pulsar (the energy is evenly distributed over the surface of a sphere of radius $\boldsymbol{r}_{\text {pul }}$ ).

A more accurate estimate will be by considering directional pattern of gravitational radiation from the pulsar.

This radiation is determined by the quadrupole moment of a source that is not known a priori. The quadrupole moment exists, if the body is not symmetrical, and therefore, in general, the GW- radiation of pulsar is not isotropic and has a significant selectivity (concentration) of the radiation in space [25]. Multiplying by directivity factor of pulsar radiation can be obtained a more accurate estimate $\Phi_{\max }\left(\omega_{p u l}, r_{p u l}\right)$ than in accordance with Equation (15).

For a signal from the registered pulsar it must be satisfied [24] [25]:

$$
\Phi_{G W}\left(\omega_{p u l}\right)<\Phi_{\max }\left(\omega_{p u l}, r_{p u l}\right) .
$$

This is a necessary condition for registration GW-radiation from real sources, this is a simple test for the reliability of detection.

Otherwise, if $\Phi_{G W}\left(\omega_{p u l}\right)>\Phi_{\max }\left(\omega_{p u l}, r_{p u l}\right)$, it means a false detection.

The total energy that gravitational wave carry past a unit surface area of detector is [16] [17]:

$$
\begin{gathered}
\Phi\left(\mathrm{erg} / \mathrm{cm}^{2}\right)=\int c \cdot T_{00}^{(G W)} \mathrm{d} t=\int \frac{\aleph}{16 \pi}\left(\dot{A}_{+}^{2}+\dot{A}_{\times}^{2}\right) \mathrm{d} t, \\
\aleph=\frac{c^{3}}{G}, \quad G=6.67 \times 10^{-8} \mathrm{~cm}^{3} /\left(\mathrm{g} \cdot \mathrm{s}^{2}\right) .
\end{gathered}
$$

Using Parseval's theorem, the energy flow per unit area [16] represented in the frequency domain:

$$
\Phi\left(\mathrm{erg} / \mathrm{cm}^{2}\right)=\int \Phi(\omega) \mathrm{d} \omega=\int \frac{\aleph \omega^{2}}{16 \pi}\left(\left|\tilde{A}_{+}(\omega)\right|^{2}+\left|\tilde{A}_{\times}(\omega)\right|^{2}\right) \mathrm{d} \omega
$$

where $A_{+}(t)=\frac{1}{\sqrt{2 \pi}} \int \tilde{A}_{+}(\omega) \mathrm{e}^{-i \omega t} \mathrm{~d} \omega, \quad A_{\times}(t)=\frac{1}{\sqrt{2 \pi}} \int \tilde{A}_{\times}(\omega) \mathrm{e}^{-i \omega t} \mathrm{~d} \omega$. 
The integrand expression in (18) is the amount of energy transferred by GW-waves per unit frequency interval and per unit area (the analogue of the equation (37.31) in [16]):

$$
\Phi_{G W}(\omega)\left(\mathrm{erg} /\left(\mathrm{cm}^{2} \cdot \mathrm{Hz}\right)\right)=\frac{\aleph \omega^{2}}{8 \pi}\left(\left|\tilde{A}_{+}(\omega)\right|^{2}+\left|\tilde{A}_{\times}(\omega)\right|^{2}\right),
$$

If the source is periodic (for example, if $A_{+}(t)=h_{+}(t)=h_{0} \sin \left(\omega_{0} t\right), h_{x}=0, \omega_{0}=2 \pi \cdot f_{0}, f_{0}$-frequency of gravitational radiation), in this case the spectral energy density of the gravitational-wave radiation is represented as:

$$
\Phi\left(\omega_{0}\right)=\frac{\aleph \omega_{0}^{2} h_{0}^{2}}{32 \pi} .
$$

Using Equations (5), (13), the spectral energy density of the gravitational-wave radiation can be represented as:

$$
\Phi\left(\omega_{0}\right)=\Phi_{G W}\left(\omega_{0}\right)=\frac{c \omega_{0}^{2} E\left(f_{0}, \boldsymbol{p}\right)}{32 \pi G} .
$$

The value of energy of the detected signal equals $E\left(f_{o}, \boldsymbol{p}\right) \approx 0.8 \times 10^{-26}(\mathrm{~m} / \mathrm{s})^{2}$ at the frequency $f_{0}=6.023$ $\mathrm{Hz}$ and according to the Equation (21):

$$
\Phi_{G W}\left(\omega_{0}\right)=5 \times 10^{-4} \mathrm{erg} /\left(\mathrm{cm}^{2} \cdot \mathrm{Hz}\right) .
$$

Relation of the radiation source with the pulsar (pulsar association) carried out by the frequency of the radiation source and position recorded on the celestial sphere [19] [25] (Briefly in Appendix A).

List of pulsars (rotation frequency $f_{\text {rot }} \approx 3 \mathrm{~Hz}, f_{\text {pul }} \approx f_{0}$ ) is shown in Table 1 [27] [28], among them the most suitable is the pulsar $\mathbf{J 0 9 4 5 - 4 8 3 3 . ~}$

Expression $\varepsilon=\Phi_{G W}\left(\omega_{0}\right) / \Phi_{\max }\left(\omega_{\text {pul }}, r_{\text {pul }}\right) \approx 3 \times 10^{-5}$ for this pulsar, thereby, test of the reliability of the detection is satisfied Equation (16).This means that $\varepsilon=3 \times 10^{-5}$ of the total rotational energy of the pulsar is spent on the gravitational radiation.

Considering heterogeneity of the gravitational radiation, it will be spent an even smaller part of the rotational energy on this radiation, i.e., $\varepsilon \leq 10^{-5}$, and it is acceptable and reasonable value of energy consumption for gravitational radiation [26].

Similarly, you can perform the detection of other sources of GW-waves.

For example, candidates for the signals can be seen near $6.12 \mathrm{~Hz}$ and $6.26 \mathrm{~Hz}, \mathrm{SNR} \approx 6.5$ and 7, respectively.

Table 1. Pulsars with rotation frequency $f_{\text {rot }} \approx 3 \mathrm{~Hz}$.

\begin{tabular}{ccc}
\hline Pulsar & $f_{\text {rot }}(\mathrm{Hz})$ & $r_{\text {pul }}, \mathrm{kpc}$ \\
\hline B0450+55 & 2.93487997706 & 1.18 \\
J0511-6508 & 3.10499386985 & 2.17 \\
J0945-4833 & 3.015812519280 & 2.71 \\
J1046+0304 & 3.06493262635 & 2.25 \\
J1604-7203 & 2.92909024976 & 2.56 \\
J1739-3951 & 2.92592314444 & 1.13 \\
B1800-27 & 2.990292674144 & 3.62 \\
J1820-0509 & 2.964537059313 & 2.81 \\
J1852-2610 & 2.97320725542 & 2.26 \\
B2020+28 & 2.912037613413 & 2.10 \\
B2048-72 & 2.92966262614 & 1.05 \\
B2123-67 & 3.0696382358 & 2.75 \\
\hline
\end{tabular}




\section{GSA Applications}

There are two types of gravitational signals: pulse and continuous quasi-harmonic.

LIGO detectors are aimed at the detection of signals of the first type [29]. GSA detects signals of the second type by prolonged accumulation.

Complementarity between LIGO and GSA is evident.

If the sensitivity of LIGO is increased to $h \approx 10^{-22}$ and more advanced methods of processing strain information are used then it is possible to detect gravitational waves from pulsars (frequency band of 35-350 Hz).

There are more than 75 pulsars of this frequency band that are located on distance less 6 kpc.

Detecting continuous signals (second type) by two methods will significantly increase the confidence of detecting gravitational waves.

By using the optimal methods of processing GSA data, 7 signals (see Appendix A) are detected with a confidence probability $P_{\text {conf }}>0.97$. These signals lie on the planes which are perpendicular to the direction of the movement on certain points of the celestial sphere and have an elliptical polarization close to $90 \%$ (eccentricity $\varepsilon>0.866$ ). The detected GSA signals are elliptically polarized, related to transverse waves and do not have longitudinal components.

Physical consistency of General Relativity or any other theory of gravity is checked for existence of a hypothetical scalar component of gravitational radiation [30]. One result of this work consists in that the transverse waves of gravitational radiation are detected and longitudinal waves are not observed.

These results support the basic provisions of General Relativity about gravitational waves:

-existence of two "canonical” polarizations $h_{+}$and $h_{\times}$;

-transverse nature of gravitational waves.

\section{Conclusions}

The required sensitivity of gravitational telescope has been achieved for the detection of gravitational waves $(h$ $\approx 10^{-21}$ ).

Relative displacements $\left(\approx 10^{-15} \mathrm{~m}\right)$ of the different points of the Earth were detected; corresponding signals lie on the planes which are perpendicular to the direction of the radiation source. These signals have a high degree of elliptical polarization.

Detection of signals for a long time (90 hours) confirms the fact of registration of the GW-wave in the frequency band near $6.023 \mathrm{~Hz}$.

Confidence probability of detection of GW-wave is close to 1.

Characteristics of detected signals (amplitude, center frequency) vary in time, i.e. GW-signal is quasi-harmonic signal with smoothly varying parameters. The source of these GW-signals is periodic (generally quasiperiodic), which is typical for pulsars.

The pulsar J0945-4833 ( $f_{\text {rot }} \approx 3.01 \mathrm{~Hz}$ ) is the most probable source of detected GW-signals, it's expended about $\varepsilon \approx 10^{-5}$ of the rotational pulsar energy on gravitational radiation.

Described universal approach can be used for the detection of pulsars with $f_{\text {rot }}>0.5 \mathrm{~Hz}$.

It is possible to increase the sensitivity of the gravitational telescope and to detect GW-waves with $h<10^{-21}$ by increasing the number of stations and accumulation intervals.

\section{References}

[1] Press, W.H. and Thorne, K.S. (1972) Gravitational-Wave Astronomy. Annual Review of Astronomy and Astrophysics, 10, 335-374. http://dx.doi.org/10.1146/annurev.aa.10.090172.002003

[2] Grishchuk, L.P., Lipunov, V.M., Postnov, K.A., Prokhorov, M.E. and Sathyaprakash, B.S. (2001) Gravitational Wave Astronomy: In Anticipation of First Sources to Be Detected. Physics-Uspekhi, 44, 1-51. http://dx.doi.org/10.1070/PU2001v044n01ABEH000873

[3] Will, C.M. (1994) The Binary Pulsar, Gravitational Waves, and the Nobel Prize. Physics-Uspekhi, 37, $697-703$. http://dx.doi.org/10.1070/PU1994v037n07ABEH000035

[4] Rudenko, V.N. (2007) Search for Gravitational Waves. VEK 2, Printing House, Fryazino, 64 p.

[5] Sadeh, D. and Meidav, M. (1972) Periodicities in Seismic Response Caused by Pulsar CP1133. Nature, 240, 36-38. http://dx.doi.org/10.1038/240136a0

[6] Rudenko, V.N. and Kravchuk, V.K. (1997) Earth as Low Frequency Gravitational Wave Detector. 
http://www.cbpf.br/ cosmogra/Escolas/8_Escola/VIIIESCOLA_438_462.pdf

[7] (1988) Physical Encyclopedia, Vol. 1. Great Russian Encyclopedia, Scientific Printing House, Moscow, 704 p.

[8] Surdin, V.G. (2011) Prospecting Distant Planets. Printing House FIZMATLIT, Moscow, 352 p.

[9] List of IMS Stations Constituting the Scope of the Contract. https://www.ctbto.org/fileadmin/user_upload/procurement/2008/RFP2009-0227-ATT_2-ROCHE.pdf

[10] Aki, K. and Richards, P.G. (1973) Quantative Seismology, Theory and Methods, Vol. 1. W.H. Freeman and Company, San Francisco, 700 p.

[11] Kedrov, O.K. (2005) Seismic Methods of Monitoring Nuclear Tests. Typography “Red October”, Moscow and Saransk, $419 \mathrm{p}$.

[12] Brune, J.F. and Oliver, J. (1959) The Seismic Noise of the Earth's Surface. Bulletin of the Seismological Society of America, 49, 349-353.

[13] Fix, J.E. (1972) Ambient Earth Motion in the Period Range from 0.01-2560s. Bulletin of the Seismological Society of America, 62, 1753-1760.

[14] Franiti, G.E., Willis, D.E. and Wilson, J.T. (1962) The Spectrum of Seismic Noise. Bulletin of the Seismological Society of America, 52, 113-121.

[15] Ashby, N. and Dreitlein, J. (1975) Gravitational Wave Reception by a Sphere. Physical Review D, 12, 336-349. http://dx.doi.org/10.1103/PhysRevD.12.336

[16] Misner, C.W., Thorne, K.S. and Wheeler, J.A. (1973) Gravitation. W.H. Freeman and Company, San Francisco, 1316 p.

[17] Jaranovski, P. and Krolak, A. (2009) Analysis of Gravitational-Wave Data. Cambridge University Press, Cambridge, 251 p. http://dx.doi.org/10.1017/CBO9780511605482

[18] Bichak, I. and Rudenko, V.N. (1987) Gravitational Waves in the General Theory of Relativity and the Problem of Their Detection. Publishing House of the Moscow State University, Moscow, 264 p.

[19] Lukanenkov, A.V. (2014) Detector of Gravitational Signals. Engineering Physics, 5, 3-15.

[20] Hewish, A. (1968) Pulsars. Scientific American, 219, 25-35. http://dx.doi.org/10.1038/scientificamerican1068-25

[21] Manchester, R.N. and Taylor, J.M. (1977) Pulsars. W.H. Freeman, San Francisco, 294 p.

[22] (1994) Physical Encyclopedia, Vol. 4. Great Russian Encyclopedia, Scientific Printing House, Moscow, 704 p.

[23] Nikolaev, A.V., Lukanenkov, A.V. and Dubrov, M.N. (2010) New Possibilities of Combined Data Processing from Recording of Displacements and Strains in the Field of Seismic Waves. Doklady Earth Sciences, 430, 258-260. http://dx.doi.org/10.1134/S1028334X10020248

[24] Zeldovich, B.Ya. and Novikov, I.D. (1971) Theory of Gravity and Evolution of Stars. Publishing House "Science", Moscow, 484 p.

[25] Lukanenkov, A.V. (2014) Experimental and Theoretical Evaluation of Detection of Gravitational Waves. Engineering Physics, 12, 42-51.

[26] Lukanenkov, A.V. (2015) Experimental Detection of Gravitational Waves. Physical Interpretation of Relativity Theory: Proceedings of International Meeting, Bauman Moscow State Technical University, Moscow, 29 June-02 July 2015, 343-358.

[27] ATNF Pulsar Catalogue. http://www.atnf.csiro.au/research/pulsar/psrcat/

[28] Manchester, R.N., Hobbs, G.B., Teoh, A. and Hobbs M. (2005) The Australia Telescope National Facility Pulsar Catalogue. The Astronomical Journal, 129, 1993-2006. http://dx.doi.org/10.1086/428488

[29] Abbott, B.P., et al. (2016) Observation of Gravitational Waves from a Binary Black Hole Merger. Physical Review Letters, 116, 061102. http://dx.doi.org/10.1103/PhysRevLett.116.061102

[30] Corda, C. (2009) Interferometric Detection of Gravitational Waves: The Definitive Test for General Relativity. International Journal of Modern Physics D, 18, 2275-2282. http://dx.doi.org/10.1142/S0218271809015904 


\section{Appendix A}

\section{The association of pulsars}

Detected signals characterized by the following parameters:

$$
f_{0}, \boldsymbol{p}_{0},\left(\varphi_{0}, \lambda_{0}\right), \boldsymbol{p}_{0}=\left(\cos \varphi_{0} \cos \lambda_{0}, \cos \varphi_{0} \sin \lambda_{0}, \sin \varphi_{0}\right) .
$$

The maximum value of $E(f, \boldsymbol{p})$ is achieved for $f=f_{0}, \boldsymbol{p}=\boldsymbol{p}_{0}$.

Pulsars, related with the detected signals, are found in a pulsar catalogue (for example, ATNF pulsar catalogue) by the rules:
1. $\frac{\left|f_{p u l}-f_{0}\right|}{f_{0}} \leq 0.03$ (3\%).

2. Right ascension $\left(\alpha_{\text {pul }}\right)$ and declination $\left(\delta_{\text {pul }}\right)$ of pulsar must be within the limits:

$\left|\alpha_{p u l}-\lambda_{0}\right|<66^{\circ},\left|\delta_{p u l}-\varphi_{0}\right|<33^{\circ}$ (These limits are due to the characteristics of the directional pattern GSA [19] [25]).

Then, pulsar is selected with rules:

Closeness in frequency $\min _{i}\left(f_{\text {pul }}(i)-f_{0}\right)$ and closeness in distance $\min _{i}\left(r_{\text {pul }}(i)\right)$ (Figure A1).

7 (seven) signals were detected from pulsars with confidence probability $P_{\text {conf }}>0.97$ according to these rules, the frequencies of signals :1.457 Hz, 1.574 Hz, 1.662 Hz, 1.896 Hz, 2.033 Hz, 3.75 Hz, 6.023 Hz [19] [25], signals were detected on the 4-hour fragment ( $T=4$ hour).

For example, signal has the characteristics $\varphi_{0}=110^{\circ}, \lambda_{0}=-60^{\circ}$ if $f_{0}=6.023 \mathrm{~Hz}$ [19] [25], and the pulsar J0945-4833 $\left(146^{\circ},-48^{\circ}\right)$ is the most suitable by the rules of association.

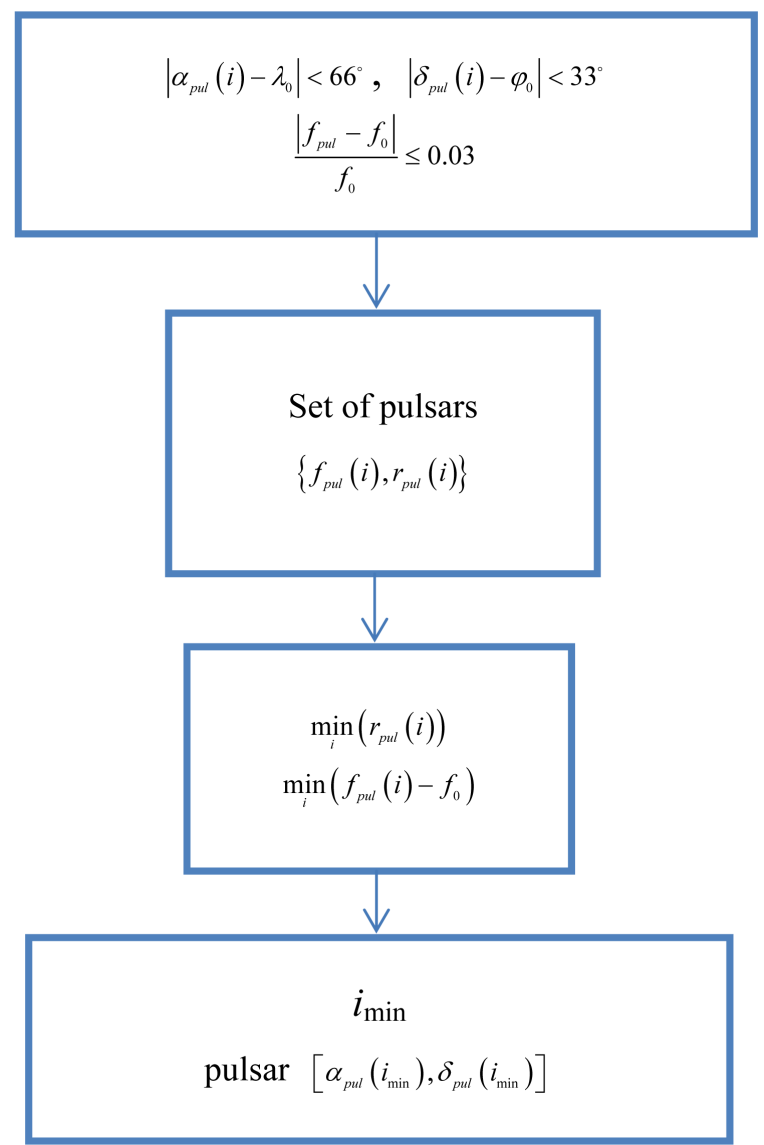

Figure A1. Diagram of pulsar association. 